Editor's Note: Toolboxes are intended to describe and evaluate methods that are becoming widely relevant to the neuroscience community or to provide a critical analysis of established techniques. For more information, see http://www.jneurosci.org/misc/ ifa_minireviews.dtl.

\title{
Imaging Living Synapses at the Nanoscale by STED Microscopy
}

\author{
U. Valentin Nägerl ${ }^{1}$ and Tobias Bonhoeffer ${ }^{2}$ \\ ${ }^{1}$ Inserm U862/Université Victor Segalen Bordeaux 2, 33076 Bordeaux, France, and ${ }^{2}$ Max Planck Institute of Neurobiology, 82152 Martinsried, Germany
}

\begin{abstract}
Introduction
Synapses are extraordinary signaling machines. Thousands of proteins precisely and uniquely arranged within the presynaptic and postsynaptic compartments of each synapse govern neuronal communication and mediate plasticity in the nervous system. One of the principle goals in neuroscience has long been to measure synaptic structure and protein organization, and to monitor mechanisms of change at individual synapses in living cells. Because synapses are very small, highly dynamic, and densely packed within light-scattering medium, this goal has been difficult to attain. Recent advances in microscopic techniques are likely to change that by improving spatial resolution by an order of magnitude, thus making it possible to investigate synaptic physiology and biochemistry at individual synapses, even at the level of single molecules.
\end{abstract}

It is along-standing experimental problem that the spatial resolution of conventional light microscopy is in the same range as the size of synapses, in particular of dendritic spines, which are tiny specializations of the postsynaptic membrane that mediate excitatory synaptic transmission in the CNS. Spines vary from 0.2 to 2 $\mu \mathrm{m}$ in length, as measured by electron microscopy (Harris and Kater, 1994), and

Received Feb, 23, 2010; revised April 17, 2010; accepted May 10, 2010.

Correspondence should be addressed to U. Valentin Nägerl, Inserm U862/Université Victor Segalen Bordeaux 2, 146 rue Léo Saignat, 33076 Bordeaux, France. E-mail: valentin.nagerl@u-bordeaux2.fr.

D01:10.1523/JNEUROSCI.0990-10.2010

Copyright $\odot 2010$ the authors $\quad 0270-6474 / 10 / 309341-06 \$ 15.00 / 0$ can thus be readily identified by conventional approaches such as confocal laser-scanning fluorescence microscopy. However, measuring changes in spine substructure, i.e., changes in spine shape and size, has been difficult because they are usually smaller than what conventional optical systems can reliably resolve. This is unfortunate given the critical role that the size and shape of synaptic structures, such as the width of spine necks, is thought to play for synaptic function. Also, the measurement of the precise location of particular proteins, such as neurotransmitter receptors and their dynamic behavior, has been very difficult due to the fact that these changes and movements often are well below $1 \mu \mathrm{m}$.

In contrast, electron microscopy provides a spatial resolution that easily resolves synapses, spines, and their substructures, but it provides no temporal information, which is crucial for many questions in synaptic physiology and plasticity. In addition, the labeling of particular proteins for electronmicroscopy is often problematic and sampling of many synapses is labor-intensive, whereas fluorescence microscopy permits live-cell imaging over relatively large volumes with biomolecular specificity.

Until a few years ago, the spatial resolution of light microscopy was thought to be fundamentally limited by a physical law put forward by Ernst Abbé over a hundred years ago, which states that the smallest detail resolvable with a light microscope is on the order of half the wavelength of the used light, i.e., 200-400 nm. New ideas and technical developments have now resoundingly shattered this resolution limit, pushing our ability to observe cellular details well below 100 nm. While not abolishing diffraction per se, which is at the heart of Abbé's theory, the new nanoscopy techniques cleverly circumnavigate its limiting effects on spatial resolution.

Stimulated emission depletion (STED) microscopy was the first concrete and implemented concept of nanoscopy, which is part of the more general RESOLFT principle [REversible Saturable OpticaL Fluorescence Transitions (Hell, 2003, 2009)]. It was recently complemented by other powerful methods based on the sequential stochastic switching of individual photoactivatable fluorophores in wide-field illumination [photoactivatable localization microscopy (PALM) and stochastic optical reconstruction microscopy (STORM)] (Betzig et al., 2006; Hess et al., 2006; Rust et al., 2006; Hell, 2009).

Although the STED principle was originally conceived some time ago (Hell and Wichmann, 1994), its practical implementation has gained substantial momentum only over the last couple of years and it is being refined at a rapid pace. STED microscopy on neurobiologically relevant topics started with studying distributions of immunohistochemically labeled proteins in fixed-tissue preparations (Kittel et al., 2006; Sieber et al., 2006; Willig et al., 2006). Its scope was then extended to monitor the movements of fluorescently labeled vesicles inside nerve terminals (Westphal et al., 2008) and to 
image activity-driven changes in the morphology of dendritic spines (Nagerl et al., 2008), using genetically encoded fluorescence (YFP) as a label.

It is thus now possible to image and capture the dynamics of dendritic spines of YFP-positive CA1 pyramidal neurons in living hippocampal slices with a resolution of better than $100 \mathrm{~nm}$. As such, it is suited for studying activitydriven structural changes of synaptic morphology, revealing a diversity of shapes and dynamics that cannot be properly quantified by diffraction-limited techniques. STED also lends itself to the study of fluorescently tagged molecules of the postsynaptic density, the cytoskeleton and the like. We have, for instance, recently used STED microscopy to image the distribution and filamentous actin inside spines with $>100 \mathrm{~nm}$ spatial resolution (V. Nägerl, unpublished observation), using fluorescently tagged lifeact, which is a small synthetic actin-binding peptide (Riedl et al., 2008).

In the following, we give a brief outline of the principles of STED microscopy and its application to a specific neurobiological context: live-cell time lapse imaging of dendritic spines of pyramidal neurons in organotypic hippocampal slices, using a genetically encoded fluorescent protein as a volume marker of the synaptic structures. In addition, we describe the basic elements needed to assemble a custombuilt STED microscope capable of livecell imaging and how to use it for physiology experiments.

\section{Imaging setup}

Principle of STED microscopy

The resolution of laser scanning microscopes (confocal and 2-photon) is limited by the fact that a laser beam-even if it was perfectly collimated-is not focused to an infinitesimally small point by the microscope's objective, but rather to a blurry intensity distribution, which is called the point-spread function (PSF) and which defines the spatial resolution of the optical system. The core idea of STED microscopy is to improve the spatial resolution by constricting the PSF beyond this classic limit (called the diffraction barrier). This is achieved by a second laser beam (called the STED beam), which can de-excite fluorescent molecules by stimulated emission at a wavelength that is longer than the fluorescence (Fig. 1A). By shaping the STED beam like a doughnut in the focal plane, it actively switches off the fluorescence on a circular rim around the PSF and thus only permits fluorescence to occur from the center of the doughnut (called the null) (Fig. $1 B$ ). As of now, the fluorescence spot size can be reduced to $\sim 10-20 \mathrm{~nm}$, which is more than an order of magnitude smaller than what diffraction-limited light microscopy can achieve. The resolution of a STED microscope is given by $\Delta r \approx \lambda /\left(2 \mathrm{NA} \sqrt{1+I / I_{\mathrm{s}}}\right)$, with NA denoting the numerical aperture of the lens, $\lambda$ the wavelength, $I$ the intensity at the crest of the doughnut-shaped STED beam, and $I_{\mathrm{s}}$ the STED intensity required to reduce the fluorescence probability of the molecules by half. The derivation of this equation is beyond the scope of this primer but has been shown previously (Harke et al., 2008).

\section{STED microscopy setup}

Figure $1 C$ shows the STED microscopy setup we used for imaging living neurons. For excitation, a pulsed laser diode (80 $\mathrm{MHz}$ pulses of $90 \mathrm{ps}$ duration at a wavelength of $485 \mathrm{~nm}$; LDH-P-C-485; Picoquant) was used. The STED light was provided by a Ti:Sapphire laser (MaiTai; Spectra-Physics) coupled to an optical parametric oscillator (OPO; APE) tuned to $598 \mathrm{~nm}$ as central wavelength. Originally of 200 fs duration, the OPO pulses are stretched by dispersion using a polarization preserving fiber (Oz Optics) of $100 \mathrm{~m}$ length to $\sim 300 \mathrm{ps}$. The expanded STED beam passes through a polymeric phase plate imprinting a helical phase delay of $\exp (i \varphi)$, with $0<\varphi<2 \pi$ (vortex phase plate; RPC Photonics) on the wave front, which produces an intensity distribution shaped like a doughnut in the focal plane of the lens (Fig. 1A). The excitation and STED laser pulses need to be precisely synchronized in time; this can be achieved by externally triggering the laser diode and setting the delay by an electronic delay generator.

A high NA objective lens (e.g., PL APO, $100 \times$, oil, NA 1.4; Leica) should be used to focus the incident light into the sample. The average focal power of the STED beam, which reduces the fluorescence by half, is on the order of 1 $\mathrm{mW}$ for YFP at a repetition rate of 80 $\mathrm{MHz}$ (measured without phase plate). By increasing the STED power above that level, the resolution can be continuously adjusted. For excitation, an average focal power of $1-10 \mu \mathrm{W}$ is sufficient, however, it can be adjusted depending on the brightness and photostability of the sample.

The epifluorescence is separated from the excitation and STED light (Fig. $1 B$ ) by a dichroic mirror (DM1). After passing through a bandpass filter for cleanup (HQ535/50; Chroma), the signal photons are collected by a photodetector, which can be a multimode optical fiber connected to an avalanche photodiode (APD; SPCM-AQR-13; PerkinElmer). When the diameter of the fiber is $\sim 80 \%$ of the back-projected Airy disc, it serves as a confocal pinhole, which conveniently blocks out light from out-of-focus planes and ambient light. In principle a pinhole is not needed, as the STED resolution improvement is accomplished by the interaction of the laser beams in the focal plane with the fluorophore.

The setup can be readily built around a commercial microscope base (upright or inverted), such as the BX61 from Olympus or the DMI 6000 from Leica, which are standard research microscopes with motorized control of the wide-field epifluorescence and the $z$-focus for convenience. In our setups, we use avalanche photodiodes for detection because their quantum yields are very high (50-60\% at $535 \mathrm{~nm}$ ) and the dark counts are negligible, although its signal response becomes significantly nonlinear at count rates larger than $2 \mathrm{MHz}$. To improve the linearity of the response for higher count rates, the dead time of the avalanche photodiode can be compensated by multiplying the measured count rate with a correction factor of $1 /\left(1-t_{\mathrm{d}} \times\right.$ $C_{\mathrm{R}}$ ), where $t_{\mathrm{d}}$ is the dead time of the $\mathrm{APD}$, which is typically in the range of 10-100 ns, and $C_{\mathrm{R}}$ is the measured count rate.

After bringing the excitation and STED PSFs into coincidence in all three spatial dimensions and synchronizing the excitation and STED pulses in time, STED images can be acquired by the following different scanning schemes: (1) stagescanning, where the sample is moved by a piezo stage (P-733; Physik Instrumente), and which is optically the most simple implementation as the laser beams stay fixed; (2) beam-scanning, where both laser beams are moved in unison over the sample by galvanometer-driven mirrors (iMIC 2030 Scan Head; Till Photonics); or (3) a combination of the two, where the beam is rapidly moved along one axis by means of a resonant mirror $(15 \mathrm{kHz}$, SC-30; EOPC), while the sample is moved more slowly along the perpendicular axis by the piezo stage (Westphal et al., 2008). Any scheme can be used in principle; the preferred choice will depend on practical issues of how well it integrates into the setup and which type 
A

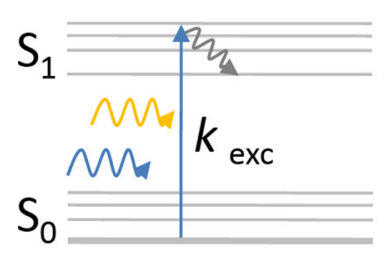

B

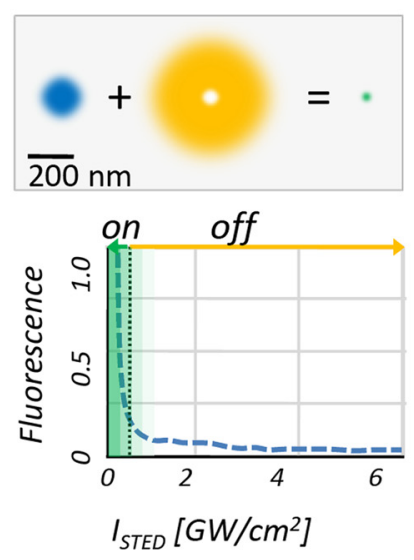

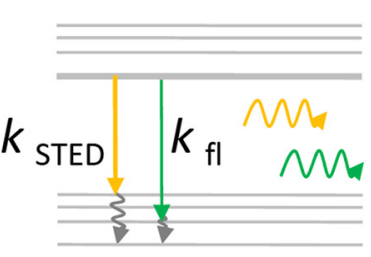

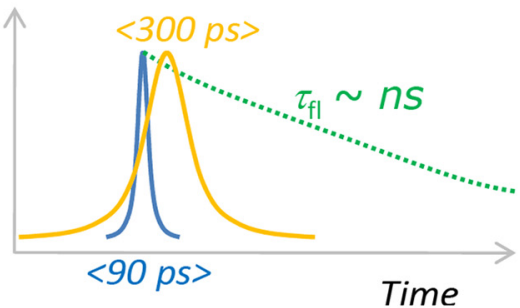

Time

C
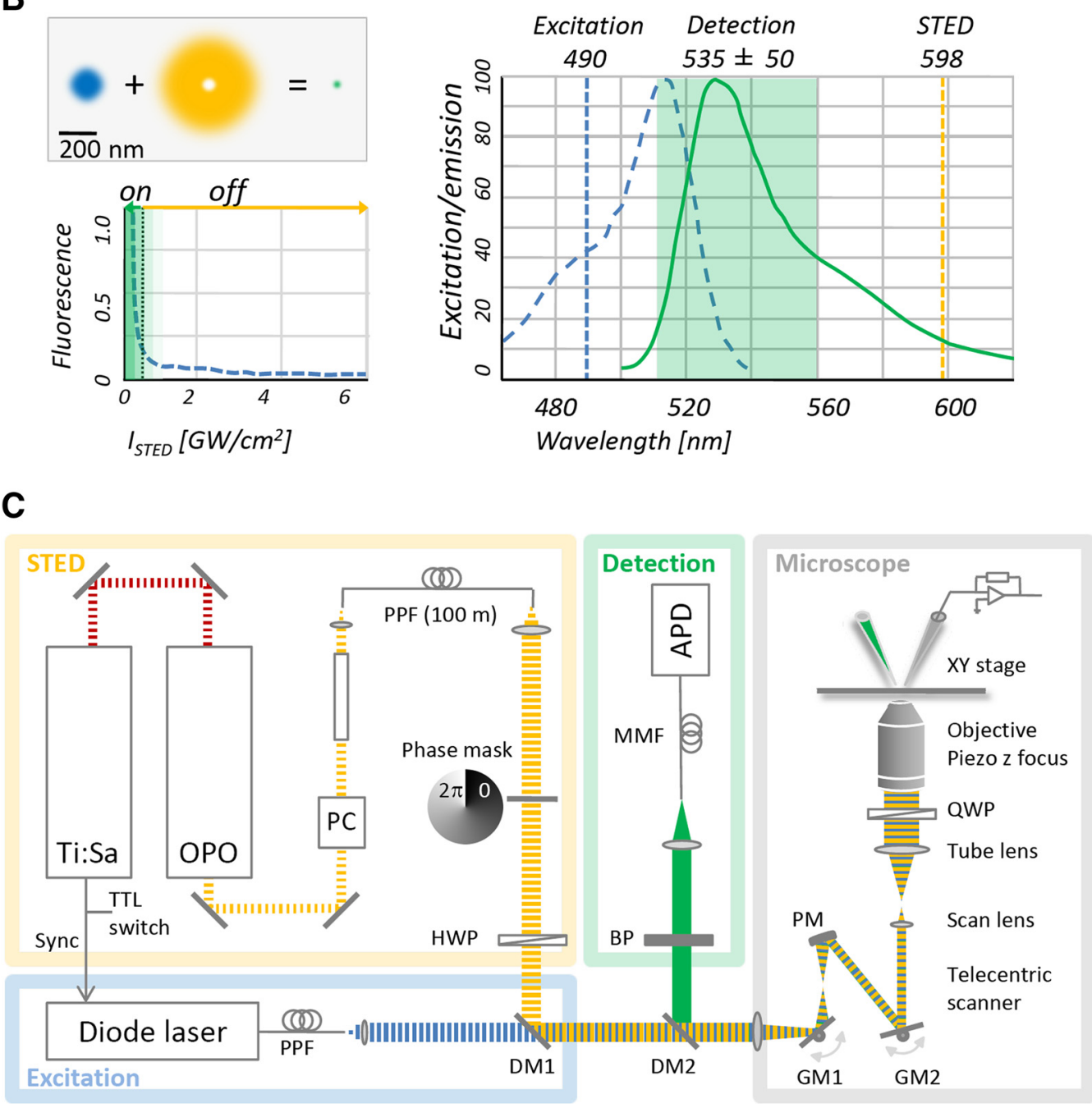

Figure 1. Principle of stimulated emission depletion microscopy. $A$, Left two panels, Jablonksi diagram of the molecular states used in STED microscopy. $k_{\text {exc }}$ denotes the photophysical transition from the ground state $S_{0}$ to the first excited state $S_{1}$ after the absorption of an excitation photon, $k_{\text {STED }}$ denotes the stimulated transition from $S_{1}$ back to $S_{0}$, and $k_{f f}$ denotes the spontaneous transition from $S_{1}$ to $S_{0}$. Right panel, Temporal relationship between excitation and STED pulses. The fluorescence lifetime $\left(\tau_{f}\right)$ of $Y F P$ is $\sim 3 \mathrm{~ns}$. $\boldsymbol{B}$, Top left panel, Superposition of the diffraction-limited spot of the excitation laser (blue) and the doughnut of the STED laser (orange) dramatically reduces the size of the fluorescence spot (green). Bottom left panel, Nonlinear relationship between the intensity of the STED beam $\left(I_{\text {STED }}\right.$ ) and the suppression of the fluorescence. The dotted line indicates the STED power that effectively turns off the fluorescence. Right panel, The wavelength of the STED beam lies at the right edge of the YFP emission spectrum, where YFP excitation is negligible. C, Optical beam path of a custom-designed STED microscope. DM, Dichroic mirror; HWP, half-wave plate; MMF, multimode optical fiber; PC, Pockels cell; PPF, polarization preserving optical fiber; QWP, quarter-wave plate; BP, bandpass filter; Ti:Sa, Ti:sapphire laser; PM, parabolic mirror; GM, galvanometric mirror.

of additional applications are intended (choice of software control, need for mounting micromanipulators etc.).

STED imaging of dendritic spines in living hippocampal slices

Hippocampal slices (300 $\mu \mathrm{m}$ thick) from YFP transgenic mice (Thyl-YFPH; prepared on postnatal days 5-7) lend them- selves for studying spines with STED microscopy, as several distinct populations of neurons, including the CAl pyramidal neurons, are strongly but sparsely labeled (Feng et al., 2000). Briefly, slices are prepared and embedded in a plasma clot on thin glass coverslips, and can be readily incubated for several weeks in a roller incubator at $35^{\circ} \mathrm{C}$, according to the Gähwiler method (Gähwiler, 1981). For the experiments, cultures are transferred to a heated chamber $\left(35^{\circ} \mathrm{C}\right)$, where they are continuously perfused with carbogenated $\left(95 \% \mathrm{O}_{2}, 5 \% \mathrm{CO}_{2}\right)$ artificial cerebrospinal fluid at $\mathrm{pH} 7.4$, containing the following (in mM): $126 \mathrm{NaCl}, 2.5 \mathrm{KCl}, 2.5$ 
$\mathrm{CaCl}_{2}, 1 \mathrm{MgCl}_{2}, 10$ glucose, $1.25 \mathrm{NaH}_{2} \mathrm{PO}_{4}$, and $26 \mathrm{NaHCO}_{3}$.

Confocal images can then be recorded by scanning the excitation beam over the sample (Figs. $2 A, B$ ). For the STED microscopy images, the STED beam is switched on additionally (Figs. 2A,B). The STED images that we recorded with this configuration had a spatial resolution of $\sim 70-80 \mathrm{~nm}$. However, because of the refractive index mismatches between the oil objective, the coverslip, and the sample, the resolution was best close to the coverslip. Despite this problem, Figure 2C clearly shows how STED imaging of spines can result in a substantial improvement of spatial resolution. It was rendered from a STED image stack recorded with a pixel size of $29 \times 29 \times 500 \mathrm{~nm}$, illustrating the potential of this technique to resolve subtle details in shape and size of living synaptic structures $\left(I_{\text {peak }}=400\right.$ $\mathrm{MW} / \mathrm{cm}^{2}$ ).

\section{Image acquisition and analysis}

Given that the resolution is greatly improved in STED imaging over conventional imaging modes, pixel and image sizes should be set accordingly. To comply with the Nyquist sampling theorem, images should be acquired with a pixel size of less than half the measured STED resolution, i.e., $30 \mathrm{~nm}$ or less. The size of the field of view will depend on which type of scanning mode is used as well as on practical issues such as frame rate or hard disk space. Although piezo stages usually do not come with large travel ranges, beamscanning certainly allows for a larger field of view. In principle, any general-purpose scanning software can be used for data acquisition and instrument control of a STED setup. However, STED microscopy requires special attention to the adjustment of the excitation-as well as the STED-beam and conventional software packages can be somewhat clumsy for this purpose. The commercially available software package "Imspector" is a useful solution to this issue, as it facilitates rapid PSF measurements (www.max-planck-innovation.de/de/ industrie/technologieangebote/software/). The choice of a custom-made or commercial software solution therefore strongly depends on the expertise and programming support available in the laboratory.

Scanning and image acquisition hardware is in principal identical to the one used for traditional scanning microscopy. Yet there is a caveat: with its substantial improvement in spatial resolution, the hardware requirements can be substan-

A
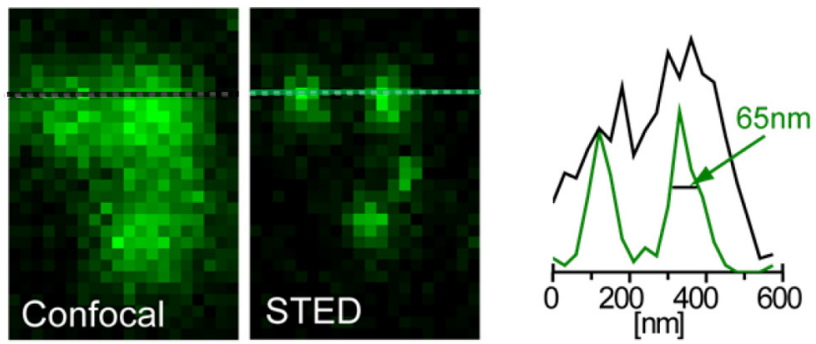

B
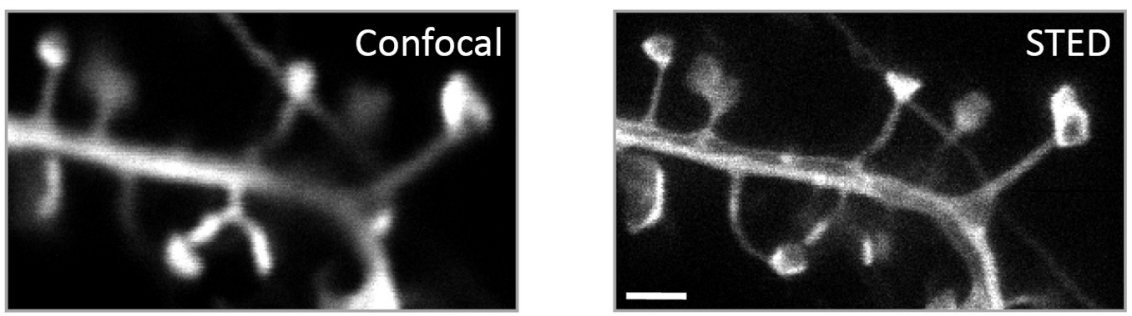

C

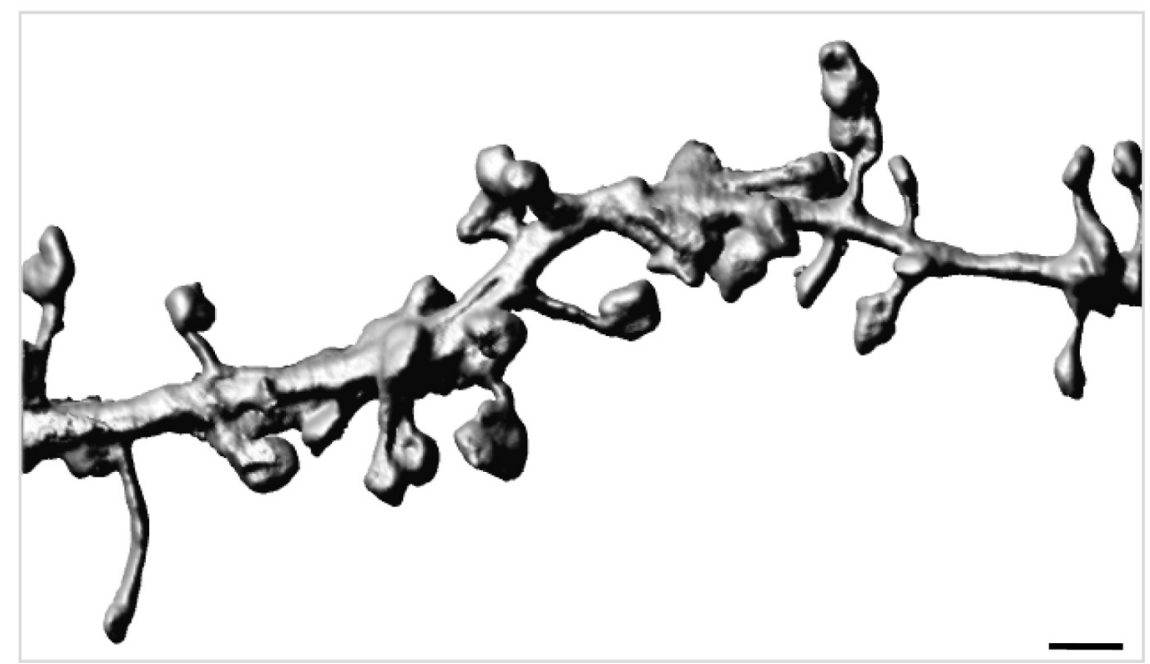

Figure 2. Breaking the diffraction barrier in synapse imaging. $\boldsymbol{A}$, Left panels, Yellow-green fluorescent beads ( $40 \mathrm{~nm}$ ) show the resolution improvement of STED versus confocal microscopy. These beads are used to check the spatial resolution of the microscope because they can be imaged with the same settings as the YFP-labeled neurons. Right panel, Plots show the intensity distribution across the dotted lines over the bead images. The full width at half maximum reduces to $65 \mathrm{~nm}$ in the STED mode, making it possible to discriminate the beads as distinct fluorescent structures. Bead images courtesy of K. Willig (Max-Planck Institute for Biophysical Chemistry, Göttingen, Germany). $\boldsymbol{B}$, Comparison of images of dendritic spines imaged either confocally or by using STED. Scale bar, $1 \mu \mathrm{m}$. C, Three-dimensional reconstruction of dendritic spines obtained by live-cell STED imaging in organotypic hippocampal slices from thy1-YFP transgenic animals (originally published in Nägerl et al., 2008). Scale bar, $1 \mu \mathrm{m}$.

tially more stringent. For instance, the electronic circuits that drive the galvanometers controlling the mirror position in a scan head are constructed such that the position noise of the mirror is substantially below the resolution limit of a 2-photon or confocal microscope and hence this noise becomes irrelevant. However, with STED microscopy providing resolution up to almost one order of magnitude better, these noise requirements are often not good enough anymore; they would degrade the STED pictures substantially. So although in general the architecture of the hardware can stay the same, the requirements in terms of electronic noise, thermal stability, etc., are substantially more stringent in a STED microscope and often components have to be exchanged or improved.

In contrast, with regard to the software to analyze images, one can fully rely on the software packages that are also used to deal with confocal images. (ImageJ, Matlab, Imaris, etc.). Finally, STED images can be further enhanced by deconvolution algorithms just like conventional confocal images, but unlike other nanoscopy techniques, image processing is, in 
principle, not needed to obtain superresolution images.

\section{Discussion}

\section{Phototoxicity and bleaching}

Phototoxicity and bleaching are, more than in conventional laser scanning microscopy, potential concerns, as the fluorophore is not only subjected to the blue excitation light, but also to the red STED laser beam, which is more intense by three orders of magnitude. Although photodamage is one of the important issues with this technique, we found that, in practice, it is possible to work with laser intensities that allow for repeated acquisition of superresolved image stacks $(>20)$ without any noticeable damage to neuronal morphology or excessive loss of signal due to bleaching.

This is likely explained by the following facts: (1) the STED beam intensity $\left(I_{\text {peak }}=400 \mathrm{MW} / \mathrm{cm}^{2}\right.$, time-averaged focal power $24 \mathrm{~mW}$ ) is still $\sim 2-3$ orders of magnitude below the intensity typically used in 2-photon microscopy (200 GW/ $\mathrm{cm}^{2}$ ) and hence also expected to cause no more damage than a 2-photon microscope, where phototoxicity and bleaching normally do not present a major problem; (2) the wavelength of the STED beam is at the red edge of the emission spectrum of the dye, producing little interaction with the dye beyond stimulated emission, which returns the fluorophore back to the nonreactive ground state. Moreover, water absorbs much less at $598 \mathrm{~nm}$ (the wavelength of the STED beam) than in the near-infrared range (by 5-20-fold); and (3) bleaching is in general less of an issue for volume-labeled structures, as the fluorescent protein (YFP) is highly expressed inside the cytosol of the neurons, where it can diffuse freely and thus replenish itself rapidly.

\section{Mechanical drift}

There are several sources of drift, which impact measurement quality over the course of the experiment. Some of them need to be suppressed a priori, others can be corrected off-line.

Mechanical drift of the optical components can greatly compromise the performance of the STED system, degrading signal strength and spatial resolution. Therefore, proper alignment of the excitation and STED laser beams should be routinely checked before the experiment (and for confirmation afterward, too). This can be readily done by using $80 \mathrm{~nm}$ reflective gold beads to measure and optimize the focal intensity distributions of the laser beams, i.e., their PSF. In addition to a solid and judicious design of the optical system (using high-quality mechanooptical components, minimizing mechanical lengths and beam paths, etc.), it is important to make sure that the temperature is kept relatively stable in the lab (varying $<1^{\circ} \mathrm{C}$ ), as thermal drift can otherwise be a problem. An interesting new design of a STED microscope effectively deals with this problem by combining the optical paths used for the excitation and STED beams. It relies on a phase plate, which selectively modulates the STED but not the excitation beam (Wildanger et al., 2009).

Sample drift that occurs between image acquisition time points is a common curse when working with living tissue, but it can usually be corrected for by aligning the images afterward using standard image registry algorithms (e.g., in ImageJ), as long as the regions of interest are fully included in all of the image stacks.

\section{STED-compatible dyes and laser sources}

A number of fluorophores have been shown to be suitable for STED microscopy; they include synthetic dyes and fluorescent proteins covering a wide spectral range (480-635 $\mathrm{nm}$ for excitation). A list of them can be viewed at www.mpibpc. mpg.de/groups/hell/STED_Dyes.html.

In principle, laser sources other than the ones outlined above are also suitable for STED microscopy. It was shown that continuous wave (CW) lasers can be used for STED microscopy of living YFPlabeled samples. They are easier to implement and maintain, and also considerably cheaper than a Ti:sapphire/OPO combination (Hein et al., 2008). However, CW STED on YFP samples requires approximately tenfold more average STED power compared with the $80 \mathrm{MHz}$ pulsed system. Ongoing developments in pulsed supercontinuum lasers promise another interesting alternative, making it possible to use a single, spectrally flexible laser source for excitation and STED, significantly reducing cost and complexity of the setup (Wildanger et al., 2008). However, current systems operate at a repetition rate of only 1-2 $\mathrm{MHz}$, which imposes relatively long recording times.

\section{Comparison with other nanoscopy techniques}

In addition to STED microscopy, other powerful techniques have recently been developed for nanoimaging of fluorescent biological samples. These rely on different theoretical principles and come with spe- cific strengths and weaknesses in terms of temporal resolution, depth penetration, multicolor imaging, practical implementation, etc. These new methods are, in theory, no longer bound by a hard resolution limit and one can achieve a resolution as high as a few nanometers under ideal conditions using beads on coverslips. However, in practice they are limited by signal noise (from drift inherent in biological samples, detector noise, etc.) to some tens of nanometers.

PALM/STORM is based on stochastic switching and computational localization of single fluorophores in wide-field illumination. Due to the single molecule nature, these techniques set the current record in spatial resolution for fluorescence microscopy, routinely achieving in biological samples a resolution better than $30 \mathrm{~nm}$ in $x-y$ and $60 \mathrm{~nm}$ in $z$ (Huang et al., 2008). Whereas temporal resolution used to be notoriously low, thanks to advances in fluorophores and image processing algorithms, acquisition times for twodimensional images can now be on the order of minutes (Wolter et al., 2010), improving PALM/STORM's suitability for imaging dynamical events inside cells. In addition, it has recently become possible to rapidly map out the movements of hundreds of particles by combining single-particle tracking with PALM. This is, however, not imaging in the true sense of the word anymore, since the tracks of a limited number of particles are localized rather than an entire image being produced. Although mostly used for imaging monolayer tissue so far, reasonable depth penetration, e.g., imaging a few cell layers below the surface of a brain slice, should in theory be possible, especially if combined with other imaging modalities, such as 2-photon laser scanning, to improve optical sectioning (Vaziri et al., 2008). PALM/ STORM can be used for 2-color imaging and its practical implementation probably is the least expensive and most straightforward among the nanoscopy techniques.

Structured illumination microscopy (SIM), another recent nanoscopy technique, relies on computationally restoring superresolved images from interferences fringes (Moiré patterns) caused by illuminating the sample with patterned light (Gustafsson, 2000). In its nonlinear variant, which requires saturating illumination of the fluorophores, a lateral resolution of $\sim 50 \mathrm{~nm}$ can be achieved by this technique (Gustafsson, 2005). Relying on wide-field imaging, the temporal resolution is quite high (on the order of 10 $\mathrm{Hz}$ ), being limited by the need to acquire 
multiple images of the Moiré patterns (10 or more). SIM poses no major constraints on imaging multiple colors, and thus it has been used for three-color imaging (Schermelleh et al., 2008), which is a distinct advantage over the other techniques.

In STED microscopy, which is a laserscanning technique like confocal or 2-photon imaging, an ensemble of fluorophores are imaged at the same time, which is very different from PALM/STORM, where single molecules must be sparsely activated at any one time. Therefore, in principle, STED is suitable for fast (on the order of kilohertz) line or point scan imaging of nanoscale domains of diffusible fluorophores, including $\mathrm{Ca}^{2+}$-sensitive dyes or other volume-labeling reporters of dynamic cellular signals. STED has been shown to work in thicker tissue sections such as brain slices (Ding et al., 2009). A combination of 2-photon excitation and pulsed STED de-excitation, as previously shown (Li et al., 2009), should extend depth penetration even further without sacrificing spatial resolution. A unique advantage of STED over other nanoscopy techniques is its potential for targeted and tunable photoactivation or uncaging at nanoscale dimensions, possibly allowing the instant creation of high concentrations of physiologically relevant molecules at predefined locations in biological tissues.

\section{Conclusions}

Nanoscopy techniques are bound to become central to neurobiological research, providing new insights into dynamic processes inside living cells at the nanoscale. As synapse function is regulated on a scale that is just a little bit too small to be resolved reliably with conventional microscopy and because mechanisms of synaptic plasticity are crucial to understanding brain function, synapses are a natural area for these new techniques. In as much as new superresolution methods have generated widespread enthusiasm for their potential to transform cell biology, it will be important to assess their scope and impact by comparing them with ongoing refinement of traditional approaches like EM and molecular biochemistry.

STED microscopy, in particular, has been shown to be suitable for live-cell imaging of molecular structures and cellular organelles at the nanoscale, using stan- dard genetically encoded fluorescence markers, such as YFP. Although STED microscopy of volume-labeled structures works particularly well, imaging lower levels of immobile fluorescence associated, for instance, with fluorescently labeled synaptic proteins is still challenging in terms of signal strength and bleaching.

Certainly, the resolving capability of STED microscopy is well established by now, and this technique has come of age in many ways, but future developments, in particular with regards to fluorescent probes and laser sources, are bound to broaden its scope and versatility and to improve the resolution even further.

\section{References}

Betzig E, Patterson GH, Sougrat R, Lindwasser OW, Olenych S, Bonifacino JS, Davidson MW, Lippincott-Schwartz J, Hess HF (2006) Imaging intracellular fluorescent proteins at nanometer resolution. Science 313:1642-1645.

Ding JB, Takasaki KT, Sabatini BL (2009) Supraresolution imaging in brain slices using stimulated-emission depletion two-photon laser scanning microscopy. Neuron 63:429-437.

Feng G, Mellor RH, Bernstein M, Keller-Peck C, Nguyen QT, Wallace M, Nerbonne JM, Lichtman JW, Sanes JR (2000) Imaging neuronal subsets in transgenic mice expressing multiple spectral variants of GFP. Neuron 28:41-51.

Gähwiler BH (1981) Organotypic monolayer cultures of nervous tissue. J Neurosci Methods 4:329-342.

Gustafsson MG (2000) Surpassing the lateral resolution limit by a factor of two using structured illumination microscopy. J Microsc 198:82-87.

Gustafsson MG (2005) Nonlinear structuredillumination microscopy: wide-field fluorescence imaging with theoretically unlimited resolution. Proc Natl Acad Sci U S A 102:1308113086.

Harke B, Keller J, Ullal CK, Westphal V, Schönle A, Hell SW (2008) Resolution scaling in STED microscopy. Opt Express 16:4154-4162.

Harris KM, Kater SB (1994) Dendritic spines: cellular specializations imparting both stability and flexibility to synaptic function. Annu Rev Neurosci 17:341-371.

Hein B, Willig KI, Hell SW (2008) Stimulated emission depletion (STED) nanoscopy of a fluorescent protein-labeled organelle inside a living cell. Proc Natl Acad Sci U S A 105:14271-14276.

Hell SW (2003) Toward fluorescence nanoscopy. Nat Biotechnol 21:1347-1355.

Hell SW (2009) Microscopy and its focal switch. Nat Methods 6:24-32.

Hell SW, Wichmann J (1994) Breaking the diffraction resolution limit by stimulated emission depletion fluorescence microscopy. Optics Lett 19:780-782.

Hess ST, Girirajan TP, Mason MD (2006) Ultra- high resolution imaging by fluorescence photoactivation localization microscopy. Biophys J 91:4258-4272.

Huang B, Wang W, Bates M, Zhuang X (2008) Three-dimensional super-resolution imaging by stochastic optical reconstruction microscopy. Science 319:810-813.

Kittel RJ, Wichmann C, Rasse TM, Fouquet W, Schmidt M, Schmid A, Wagh DA, Pawlu C, Kellner RR, Willig KI, Hell SW, Buchner E, Heckmann M, Sigrist SJ (2006) Bruchpilot promotes active zone assembly, Ca2 + channel clustering, and vesicle release. Science 312:1051-1054

Li Q, Wu SS, Chou KC (2009) Subdiffractionlimit two-photon fluorescence microscopy for GFP-tagged cell imaging. Biophys J 97:3224-3228

Nägerl UV, Willig KI, Hein B, Hell SW, Bonhoeffer T (2008) Live-cell imaging of dendritic spines by STED microscopy. Proc Natl Acad Sci U S A 105:18982-18987.

Riedl J, Crevenna AH, Kessenbrock K, Yu JH, Neukirchen D, Bista M, Bradke F, Jenne D, Holak TA, Werb Z, Sixt M, Wedlich-Soldner R (2008) Lifeact: a versatile marker to visualize F-actin. Nat Methods 5:605-607.

Rust MJ, Bates M, Zhuang X (2006) Subdiffraction-limit imaging by stochastic optical reconstruction microscopy (STORM). Nat Methods 3:793-795.

Schermelleh L, Carlton PM, Haase S, Shao L, Winoto L, Kner P, Burke B, Cardoso MC, Agard DA, Gustafsson MG, Leonhardt H, Sedat JW (2008) Subdiffraction multicolor imaging of the nuclear periphery with 3D structured illumination microscopy. Science 320:1332-1336.

Sieber JJ, Willig KI, Heintzmann R, Hell SW, Lang $\mathrm{T}$ (2006) The SNARE motif is essential for the formation of syntaxin clusters in the plasma membrane. Biophys J 90:2843-2851.

Vaziri A, Tang J, Shroff H, Shank CV (2008) Multilayer three-dimensional super resolution imaging of thick biological samples. Proc Natl Acad Sci U S A 105:20221-20226.

Westphal V, Rizzoli SO, Lauterbach MA, Kamin D, Jahn R, Hell SW (2008) Video-rate farfield optical nanoscopy dissects synaptic vesicle movement. Science 320:246-249.

Wildanger D, Rittweger E, Kastrup L, Hell SW (2008) STED microscopy with a supercontinuum laser source. Opt Express 16:96149621.

Wildanger D, Bückers J, Westphal V, Hell SW, Kastrup L (2009) A STED microscope aligned by design. Opt Express 17:16100-16110.

Willig KI, Rizzoli SO, Westphal V, Jahn R, Hell SW (2006) STED microscopy reveals that synaptotagmin remains clustered after synaptic vesicle exocytosis. Nature 440:935-939.

Wolter S, Schüttpelz M, Tscherepanow M, van de Linde S, Heilemann M, Sauer M (2010) Real-time computation of subdiffractionresolution fluorescence images. J Microsc 237: $12-22$. 